\title{
The Role of Weak Interactions in the Phase Transition and Distinct Mechanical Behavior of Two Structurally Similar Caffeine Co-crystal Polymorphs Studied by Nanoindentation
}

\author{
Soumyajit Ghosh, ${ }^{\dagger}$ Arobendo Mondal, ${ }^{\dagger}$ M. S. R. N. Kiran, ${ }^{\ddagger}$ U. Ramamurty, ${ }^{*},{ }^{\ddagger}$, and C. Malla Reddy ${ }^{*}{ }^{\dagger}$ \\ ${ }^{\dagger}$ Department of Chemical Sciences, Indian Institute of Science Education and Research Kolkata, 741252, Nadia, West Bengal, India \\ ${ }^{\ddagger}$ Department of Materials Engineering, Indian Institute of Science, Bangalore-560012, India \\ ${ }^{\S}$ Center of Excellence for Advanced Materials Research, King Abdulaziz University, Jeddah 21589, Saudi Arabia
}

\section{Supporting Information}

\begin{abstract}
Although weak interactions, such as $\mathrm{C}-\mathrm{H} \cdots \mathrm{O}$ and $\pi$ stacking, are generally considered to be insignificant, it is their reorganization that holds the key for many a solid-state phenomenon, such as phase transitions, plastic deformation, elastic flexibility, and mechanochromic luminescence in solid-state fluorophores. Despite this, the role of weak interactions in these dynamic phenomena is poorly understood. In this study, we investigate two co-crystal polymorphs of caffeine:4-chloro-3-nitrobenzoic acid, which have close structural similarity (2D layered structures), but surprisingly show distinct mechanical behavior. Form $\mathbf{I}$ is brittle, but shows shearinduced phase instability and, upon grinding, converts to Form II, which is soft and plastically shearable. This observation is in contrast to those reported in earlier studies on aspirin, wherein the metastable

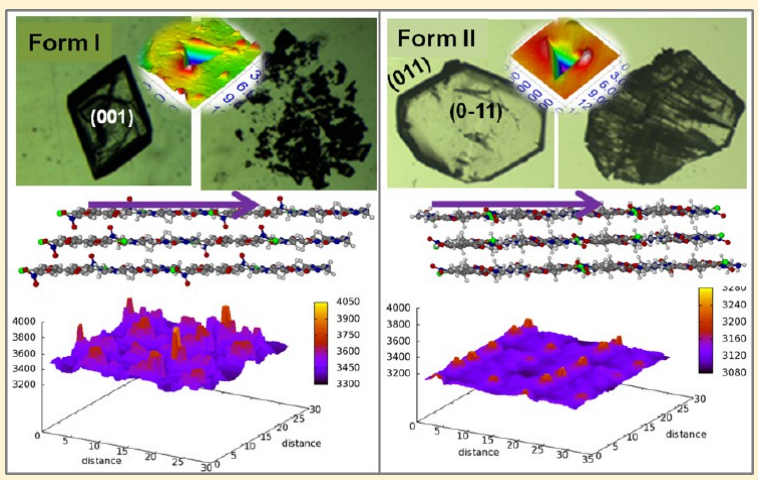
drug forms are softer and convert to stable and harder forms upon stressing. To establish a molecular-level understanding, we have investigated the two co-crystal polymorphs I and II by single-crystal X-ray diffraction, nanoindentation to quantify mechanical properties, and theoretical calculations. The lower hardness (from nanoindentation) and smooth potential surfaces (from theoretical studies) for shearing of layers in Form II allowed us to rationalize the role of stronger intralayer $\left(\mathrm{sp}^{2}\right) \mathrm{C}-\mathrm{H} \cdots \mathrm{O}$ and nonspecific interlayer $\pi$-stacking interactions in the structure of II. Although the Form I also possesses the same type of interactions, its strength is clearly opposite, that is, weaker intralayer $\left(\mathrm{sp}^{3}\right) \mathrm{C}-\mathrm{H} \cdots \mathrm{O}$ and specific interlayer $\pi$-stacking interactions. Hence, Form I is harder than Form II. Theoretical calculations and indentation on (111) of Form I suggested the low resistance of this face to mechanical stress; thus, Form I converts to II upon mechanical action. Hence, our approach demonstrates the usefulness of multiple techniques for establishing the role of weak noncovalent interactions in solid-state dynamic phenomena, such as stress-induced phase transformation, and hence is important in the context of solid-state pharmaceutical chemistry and crystal engineering.
\end{abstract}

\section{INTRODUCTION}

The understanding of structure-property relationships in molecular crystals mandates the determination of the precise roles played by various noncovalent interactions in a fairly complex structural organization. ${ }^{1,2}$ This is particularly true in the case of mechanical response ${ }^{3}$ of crystals, wherein the weak interactions play a vital role. Note that the weakest links, and not the strongest ones, often determine the strength of a solid. ${ }^{4 a}$ Although interactions, such as $\mathrm{C}-\mathrm{H} \cdots \mathrm{O}$ and $\pi$-stacking, are generally considered to be insignificant, their reorganization is the key to many a solid-state phenomenon, such as phase transitions, mechanical detonation of explosives, plastic deformation, elastic flexibility, and mechanochromic luminescence. ${ }^{4}$ The understanding of coordinated molecular movements in crystals also has implications for fatigue-resistant flexible organic electronic materials, mechanical actuators, etc. ${ }^{5}$ Hence, the weak interactions are being increasingly probed so to understand their precise role in complex structural organization in the broad framework of crystal engineering. In this context, polymorphism ${ }^{6}$ exhibited by some compounds can be exploited, as it can reduce the complexity considerably because the molecular structure is the same irrespective of the crystal structure. In addition, in some cases, the polymorphs with close structural resemblance facilitate a direct correlation of the structural differences with the measured properties. Although polymorphic phase transitions are common among active pharmaceutical ingredients (APIs) during various production processes ${ }^{7}$ (milling, grinding, handling, and tabletting, for example), the rearrangement of intermolecular interactions and local molecular movements associated with

Received: June 21, 2013

Revised: August 16, 2013

Published: August 26, 2013 


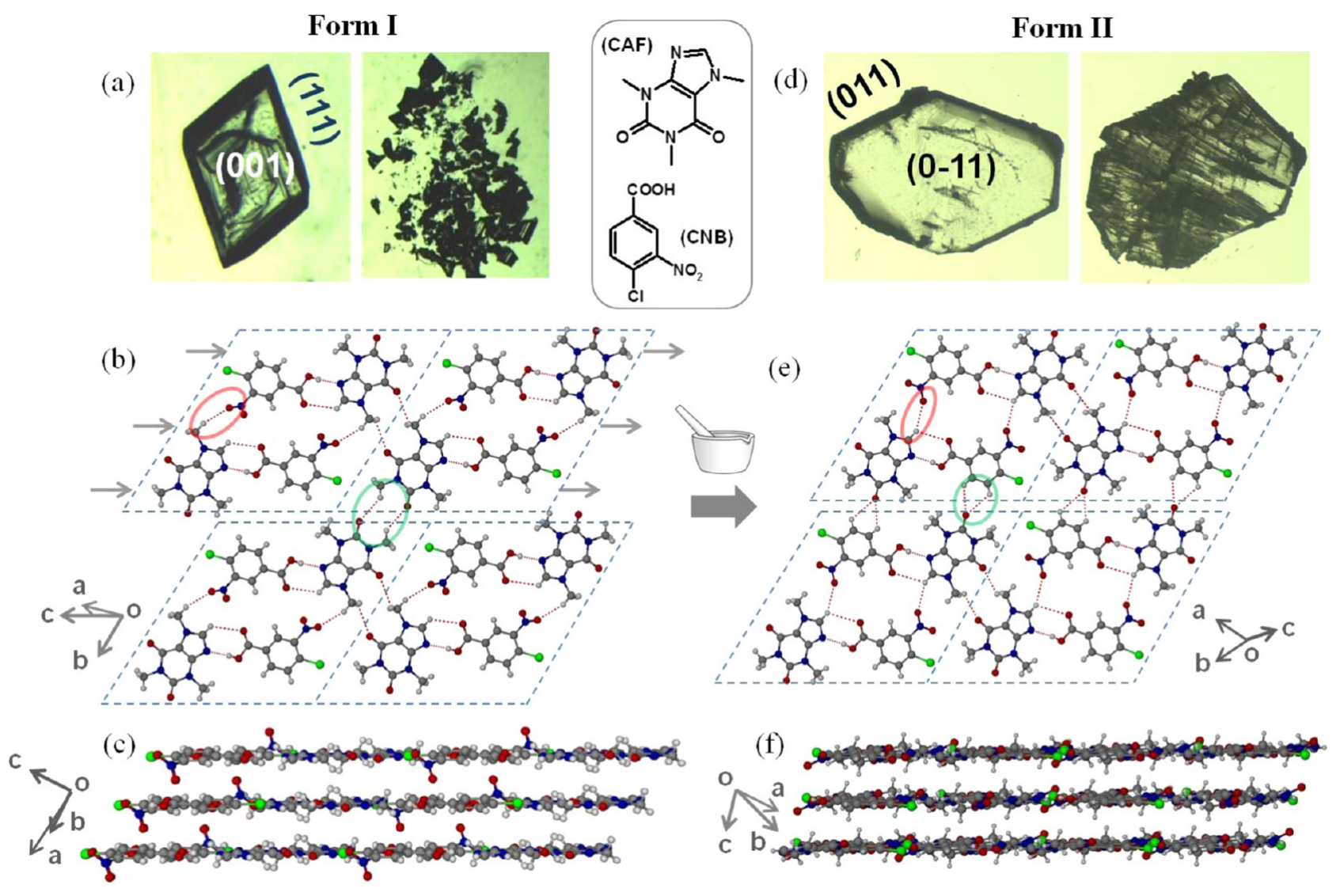

Figure 1. (a) Brittle fracture of crystals, (b) interactions in 2D layer packing, and (c) side view of stacked layers parallel to (21 $\overline{2})$ in Form I. (d) Shearing of crystals, (e) interactions in 2D layer packing, and (f) side view of stacked layers parallel to (123) in Form II. Molecular structures of the two coformers, CAF and CNB, are in the inset. Blue dotted parallelograms in (b) and (e) denote the tetramers. The conversion of Form I to Form II upon mechanical grinding requires the movement of the top two tetramers in (b) in the direction of gray arrows and the reorganization of $\mathrm{C}-\mathrm{H} \cdots$ O interactions, marked with red and green circles. The slip traces on the sheared crystal in (d) (right) (higher magnification image in Figure S9, Supporting Information) run parallel to the slip planes (123) between 2D layers. This observation suggests these crystals as $2 \mathrm{D}$ layered structures rather than stacked columns. ${ }^{4 c, 15}$

these events during mechanical loading remains poorly understood.

\section{EXPERIMENTAL SECTION}

Materials. Caffeine and 4-chloro-3-nitrobenzoic acid were purchased from Sigma-Aldrich. Commercially available solvents were used as received without further purification.

Single Crystal Preparation. Caffeine and co-crystal former in a definite stoichiometric ratio $(1: 1)$ were subjected to grinding with the addition of a few drops of methanol solvent using an agate mortar and pestle for about $15 \mathrm{~min}$. After grinding, the solid was transferred to a $10 \mathrm{~mL}$ conical flask and dissolved thoroughly in hot methanol. The resulting clear solution was filtered into a fresh conical flask and left for solvent slow evaporation at ambient conditions. Both the Forms I (light yellow, regular prisms) and II (yellow irregular blocks) grow concomitantly from methanol by slow evaporation. A third form, methanol solvate of this co-crystal, also occasionally grows concomitantly under the same conditions. ${ }^{4 \mathrm{~d}}$ The single crystals suitable for X-ray diffraction studies were obtained in 4 to 6 days.

Single-Crystal X-ray Diffraction (SCXRD). Two polymorphs of caffeine co-crystals were individually mounted on a glass pip. Intensity data were collected on a Brukar's KAPPA APEX II CCD Duo system with graphite-monochromatic Mo $\mathrm{K} \alpha$ radiation $(\lambda=0.71073 \AA)$ at 100 K. Data reduction was performed using Bruker SAINT software. ${ }^{8}$ Crystal structures were solved by direct methods using SHELXL-97 and refined by full-matrix least-squares on $F^{2}$ with anisotropic displacement parameters for non-H atoms using SHELXL-97. Hydrogen atoms associated with carbon atoms were refined in geometrically constrained riding positions. Hydrogen atoms associated with oxygen and nitrogen atoms were included in the located positions. Crystallographic data and structure refinement parameters are included in Tables S1 and S2 (Supporting Information). Structure graphics shown in the figures were created using the X-Seed software package version 2.0. ${ }^{9}$

Nanoindentation. Crystal faces of all the samples were identified and marked based on face indexing using SCXRD. The selected crystals of Forms I and II were firmly mounted on a sample holder using a very thin layer of cyanoacrylate glue prior to nanoindentation. Indentation experiments were conducted on (001) and (111) faces of I and $(0 \overline{1} 1)$ and (011) faces of II using a nanoindenter (Triboindenter of Hysitron, Minneapolis, MN) with an in situ imaging capability. The machine continuously monitors and records the load, $P$, and displacement, $h$, of the indenter with force and displacement resolutions of $1 \mathrm{nN}$ and $0.2 \mathrm{~nm}$, respectively. A three-sided pyramidal Berkovich diamond indenter (tip end radius $\sim 100 \mathrm{~nm}$ ) was used to indent the crystals. In all cases, loading and unloading rates of $0.6 \mathrm{mN} /$ $\mathrm{s}$ and a hold time of $30 \mathrm{~s}$ at peak load were employed. To identify flat regions for the experiment, the crystal surfaces were imaged prior to indentation using the same indenter tip. A minimum of 10 indentations were performed on each crystallographic face. The indentation impressions were captured immediately after unloading so as to avoid any time-dependent elastic recovery of the residual impression. The $P-h$ curves obtained were analyzed using the standard Oliver-Pharr method ${ }^{10,11}$ to extract the elastic modulus, $E$, and hardness, $H$, of the crystal in that orientation. 


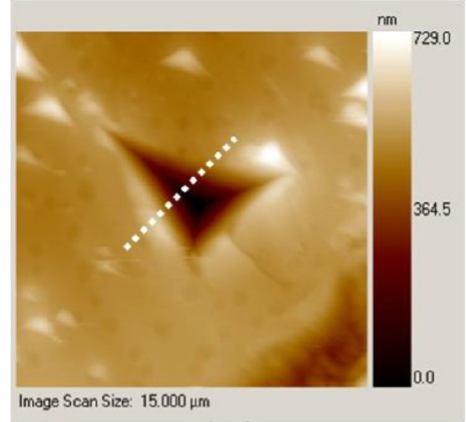

(a)

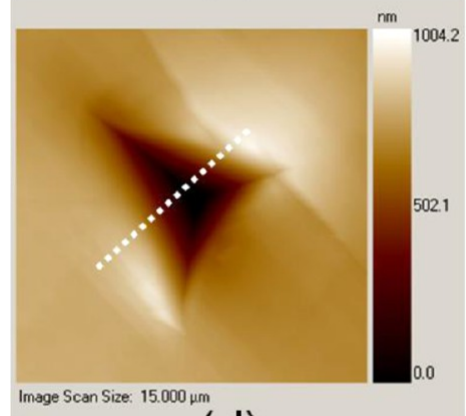

(d)

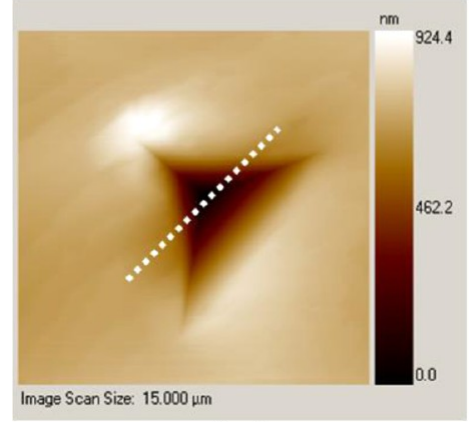

(g)

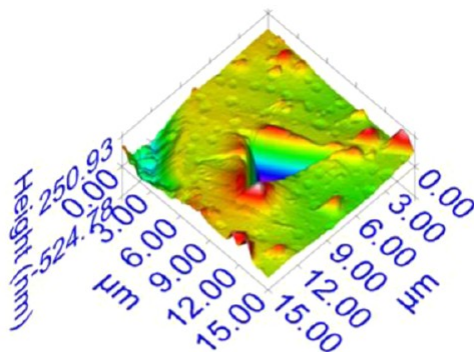

(b)

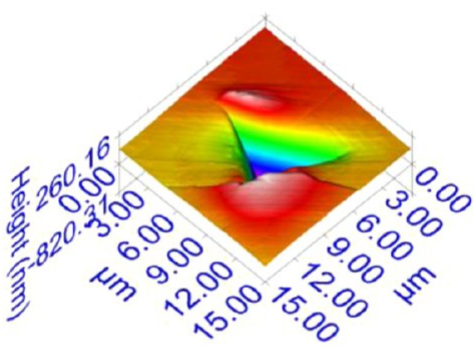

(e)

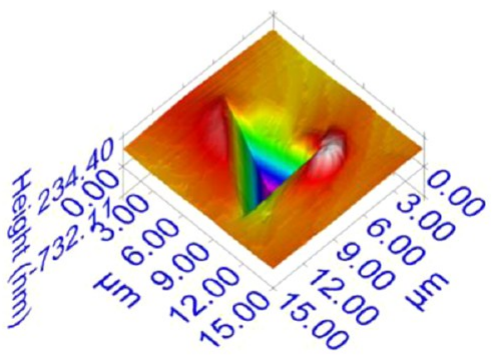

(h)

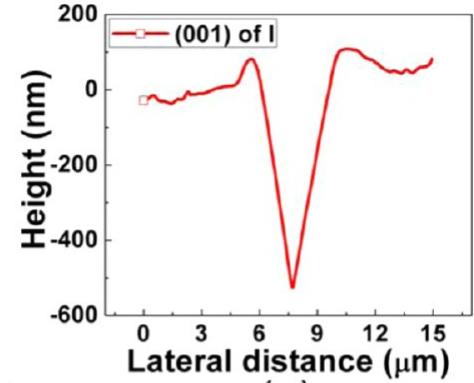

(c)

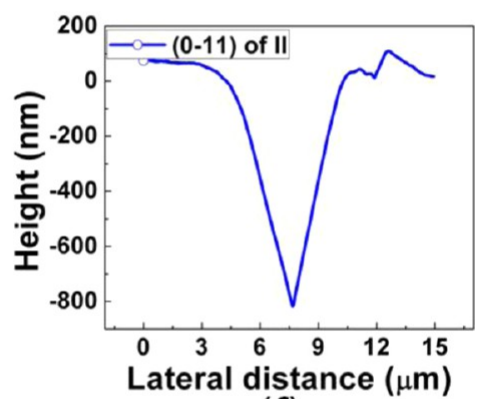

(f)

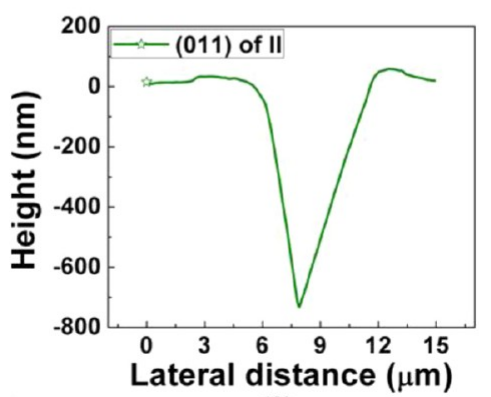

(i)

Figure 2. AFM images, 3D representations, and the corresponding line profiles at the middle of the residual indent impressions of (a-c) (001) of I, and $(\mathrm{d}-\mathrm{f})(0 \overline{1} 1)$ and $(\mathrm{g}-\mathrm{i})(011)$ of II. The dotted lines on the 2D AFM scans represent the positions where the line profiles were drawn.

\section{RESULTS AND DISCUSSION}

In this study, we investigated two structurally similar polymorphs I and II of a 1:1 co-crystal between caffeine, $\mathrm{CAF}$, and 4-chloro-3-nitrobenzoic acid, CNB, (inset, Figure 1) using single-crystal X-ray diffraction (SCXRD), quantitative mechanical property measurements using the nanoindentation technique, and complementary theoretical calculations. The nanoindentation technique, ${ }^{12}$ which is now a well-established means for understanding supramolecular bonding, ${ }^{3 a-e}$ has provided us the experimental evidence that correlates the elastic moduli and hardness of the two polymorphs to the underlying structural differences.

The crystals of Form I show mechanical instability and convert to Form II upon neat grinding for about $1 \mathrm{~h}$ (Figures S1 and S2, Supporting Information). When the macroscopic mechanical behavior of both the crystals was examined by simple and qualitative mechanical tests using a metal needle and forceps, Form I was found to be harder and brittle (Figure 1a) than Form II, which undergoes plastic shear deformation (Figure 1d, Figure S9, Supporting Information). This is somewhat surprising and counterintuitive, as earlier studies indicated that metastable forms are softer and convert to stable and harder forms upon stressing, for instance, the dimorphs of aspirin $(\text { II } \rightarrow \text { I) })^{3 a}$ venlafixin hydrochloride (Form $2 \rightarrow$ hydrate $){ }^{13}$ paracetamol $(\mathrm{II} \rightarrow \mathrm{I})$, etc. ${ }^{14}$ To gain more insights, we have investigated the caffeine polymorphs I and II in detail.

Results of SCXRD reveal that both the forms belong to the triclinic $P \overline{1}$ space group with one molecule of each coformer in the asymmetric unit, which form dimers via a robust carboxylic acid- $\mathrm{N}_{\text {(imidazole) }}$ synthon (Figure $1 \mathrm{~b}, \mathrm{e}$ ). The molecules in both the structures adopt 2D layer packing (Figure 1). Two adjacent dimers, arranged laterally with centrosymmetry, are connected via two $\mathrm{C}-\mathrm{H} \cdots \mathrm{O}$ interactions $\left(\mathrm{sp}^{3} \mathrm{C}-\mathrm{H}\right.$ in $\mathrm{I}$; $\mathrm{sp}^{2} \mathrm{C}-\mathrm{H}$ in II) and thus form tetramers. These tetramers interact via another set of $\left(\mathrm{sp}^{3}\right) \mathrm{C}-\mathrm{H} \cdots \mathrm{O}$ hydrogen bonds to form $1 \mathrm{D}$ tapes. These tapes are further packed antiparallelly to each other, leading to the $2 \mathrm{D}$ sheet structure in both the forms. The $2 \mathrm{D}$ sheets are stabilized by $\pi$-stacking interactions with the layer separation being 3.271 and $3.398 \AA$ for Forms I and II, respectively. Other than these $\pi$-stacking interactions, there are no H-bonding interactions between layers in both Forms. Prima facie, the major difference between the two structures lies in the nature of $\mathrm{C}-\mathrm{H} \cdots \mathrm{O}$ and $\pi$-stacking interactions. In Form $\mathrm{I}$, the $\mathrm{C}-\mathrm{H} \cdots \mathrm{O}$ interactions, within the tetramers (red circle, Figure $1 \mathrm{~b}$ ) and 
between the $1 \mathrm{D}$ tapes (green circle, Figure $1 \mathrm{~b}$ ), are formed from the less acidic, aliphatic $\left(\mathrm{sp}^{3}\right) \mathrm{C}-\mathrm{H}$ groups, whereas, in Form II, these are more acidic, aromatic $\left(\mathrm{sp}^{2}\right) \mathrm{C}-\mathrm{H}$ groups (Figure 1e). As a result, the 2D layers in Form II are relatively tighter than those in Form I, which, in turn, force the nitro group of CNB molecules to be coplanar with the $2 \mathrm{D}$ sheet (see Figure $1 \mathrm{c}, \mathrm{f}$ ). They also prevent optimized interlayer $\pi$-stacking interactions in II, as evident from its larger layer separation, $3.398 \AA$ vis-á-vis that of $3.271 \AA$ in I.

As a result of this suboptimal organization, shear sliding of molecules in II can be expected to be easier than that in I through the low resistance slip of $2 \mathrm{D}$ layers. Although this rationalizes the relatively softer nature of II, it fails to explain the mechanical instability of Form I. To further ascertain this, we carried out (i) nanoindentation experiments to quantify the mechanical properties - and the anisotropy in them-of both the forms and (ii) theoretical calculations for a qualitative understanding of the phase transformation and mechanical shearing, by considering the possible local molecular movements in both the structures.

Nanoindentation experiments were conducted on (001) and (111) faces of I and (011) and (011) faces of II. Atomic force microscopic (AFM) images of indentations made on various faces are shown in Figure 2. Average values of elastic moduli $(E)$ and hardness $(H)$ extracted using standard methods from the load $(P)$ versus depth $(h)$ curves displayed in Figures 3 and

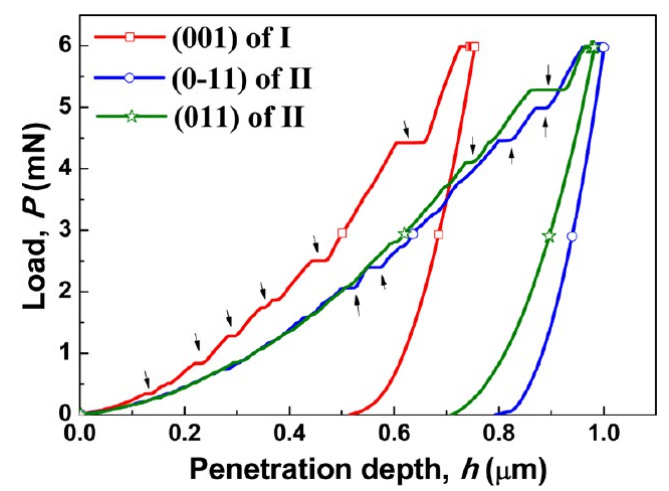

Figure 3. Representative $P-h$ curves from crystals of Form I, obtained with indentation normal to (001), and the Form II of $(0 \overline{1} 1)$ and (011) planes. Arrows indicate discrete displacement bursts or pop-ins.
4 are listed in Table 1 . The indents made on the (111) face of I showed substantial pile-up and hence could not be analyzed for

Table 1. $E$ and $H$ of the Faces of Forms I and II

\begin{tabular}{cccc} 
polymorph & orientation & $E(\mathrm{MPa})$ & $H(\mathrm{MPa})$ \\
Form I (brittle) & $(001)$ & $7860 \pm 28$ & $175 \pm 5$ \\
& $(111)$ & & \\
Form II (shear) & $(0 \overline{1} 1)$ & $6715 \pm 9$ & $106 \pm 3$ \\
& $(011)$ & $4920 \pm 5$ & $96 \pm 3$ \\
\hline
\end{tabular}

$E$ and $H$ with reasonable accuracy (see Figure 4). Data in Table 1 show that both $E$ and $H$ of II, of both the faces examined, are much lower than the respective values of the (001) face of $\mathbf{I}$. This confirms that it is relatively easy to deform II than I, which is in good agreement with the results of the macroscopic mechanical behavior as well as their crystal structures.

The $P-h$ curves on (001) of I and (0 $\overline{1} 1)$ and (011) of II show several discrete displacement bursts ${ }^{16-18}$ ("pop-ins") in the loading portion, indicating that the plastic deformation in both the crystals is intermittent in nature. A statistical analysis of the magnitudes of the displacement $(\Delta h)$ associated with the pop-ins (see Table S3, Supporting Information) clearly demonstrates that, in all the three cases, $\Delta h$ is an integral multiple of the unit cell dimension along the indentation axis. ${ }^{3 \mathrm{a}-\mathrm{d}}$ Such a correspondence was also noted in other organic crystals, such as aspirin $^{3 \mathrm{a}}$ and saccharin, ${ }^{3 \mathrm{~d}}$ and has been correlated with the intermittent plastic flow due to sudden and collective slip of crystallographic planes, which occurs so as to relax the indentation-imposed stress. Indentations on the major face of I show some pile-up, a sign of plastic flow, along the sides of the indenter. In contrast, no significant pile-up was found on either faces of II. Instead, a number of slip lines are seen, indicating that the plastic deformation is due to planar slip.

In contrast, indentation on (111) shows extremely low resistance to the penetration of the indenter, a smooth $P-h$ curve (i.e., no pop-ins), and substantial pile-up around the indents (Figure 4). Note that the loading part of the $P-h$ curve displayed in Figure 4a has two distinct segments. The initial part $(0$ to $\sim 1.5 \mu \mathrm{m}$ ) shows considerably low resistance to flow. For comparison, $P$ is only about $0.25 \mathrm{mN}$ at $h=0.5 \mu \mathrm{m}$, whereas it is $\sim 2 \mathrm{mN}$ or more at the same $h$ for all the other face indentations (on both Forms I and II) whose $P-h$ responses are displayed in Figure 3. The initial ultrasoft $P-h$ response is

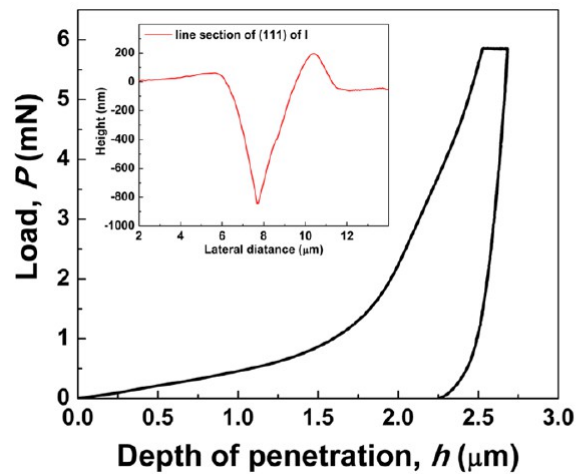

(a)

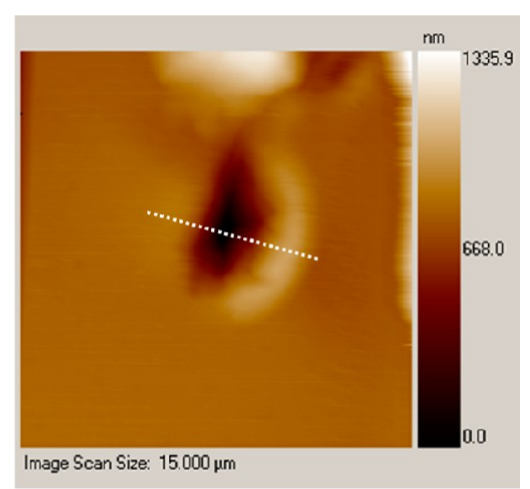

(b)

Figure 4. (a) Representative $P-h$ curve of (111) of Form I. (b) An AFM mage of the indent, which shows an irregularly shaped impression. Inset in (a) shows the line profile, drawn across the impression (dotted line) shown in (b). 
followed by a considerably steep increase in $P$ at $h$ beyond $\sim 1.8$ $\mathrm{mm}$. This apparent "hardening" is most likely due to the high amount of pile-up, as can be seen from both Figure $4 \mathrm{~b}$ and the line scan shown in the inset in Figure 4a. Again, compare the pile-up heights here to those displayed in Figure 2c,f,i. Recall that all the indentations were performed to the same maximum load, $P_{\max }$ of $6 \mathrm{mN}$. The pile-up height on (111) of Form $\mathbf{I}$ is $\sim 200 \mathrm{~nm}$, whereas it is only about $100 \mathrm{~nm}$ on (001) of I (Figure 2c) and much less (of the order of a few tens of nanometers) in the case of indentation on both the faces of II (Figure 2f,i). Such a large pile is the cause for the irregularly shaped indent. In addition, it precludes the possibility of extracting $E$ and $H$ values from the indentations made on (111) of I. An important observation to make from the $P-h$ curve displayed in Figure $4 \mathrm{a}$ is the absence of pop-ins on this face (unlike the other faces in both I and II). This indicates that the plastic flow is continuous (and not intermittent as in the other faces) in addition to being extremely easy. Hence, it is suggested that this is most probably due to the stress-induced phase transformation from Form I to II.

A comparison with the $P-h$ curves displayed in Figures 3 and 4 clearly indicates that this facet is the softest among all the four cases examined. This is because the indentation direction on this face is nearly parallel to the direction of suggested molecular shearing for the I $\rightarrow$ II phase transformation (see Figure 1b). Thus, stress-induced phase transformation is facilitated by the easy glide of molecular layers; this is the reason for the absence of the pop-ins in the $P-h$ curves. On the other hand, all the other three faces are not amenable to such an easy glide, and hence, plastic flow is difficult and intermittent. Ready plastic flow of the material, which is incompressible in nature, is the cause for the large pile-up of it against the indenter faces on (111). Importantly, no plastic deformation at the macroscopic level is seen in Form $\mathbf{I}$; instead, the crystals break in a brittle manner. A probable reason for the failure of the microscopic plasticity, observed during nanoindentation on (111), to translate into macroscopic ductility is the following. The easy shearing observed in the initial stages of plastic deformation is facilitated by the structural phase transformation. Such a situation is favored only in contact loading conditions, which prevail during the early stages of indentation or during grinding, wherein repeated local loading occurs. However, such deformation gets readily saturated as large-scale molecular motion, spanning the entire crystal's thickness, is energetically too expensive. Rapid hardening seen during the loading part of the $P-h$ curve at higher depths (Figure 4) clearly indicates to this; in fact, the hardening rate $(\mathrm{d} P / \mathrm{d} h)$ in this stage is much higher than that seen during loading on both the faces of Form II (Figure 3). Hence, Form I is macroscopically brittle, whereas, in contrast, Form II, which can facilitate such larger molecular movements by sliding of layers, is ductile. The identification of such stress sensitivity in different forms of a crystalline solid may have implications to handling of APIs in the pharmaceutical industry. For instance, the stability of a metastable form may be improved by selective suppression of the growth of mechanically sensitive crystal faces during crystallization, for example, by controlling the crystal morphology by changing solvent or using additives. ${ }^{19}$

To further understand the stress-induced instability of Form I and its phase transformation to Form II, we conducted a theoretical study at the AM1 semiempirical level using the Gaussian 03 package. Crystal structures of Forms I and II displayed in Figure 1 suggest that the conversion of Form $\mathbf{I}$ to
II requires (i) the translation of alternate $1 \mathrm{D}$ tapes along the tape direction and (ii) a slight rearrangement of molecules within the $1 \mathrm{D}$ tapes (Figure 5). Each of the above two

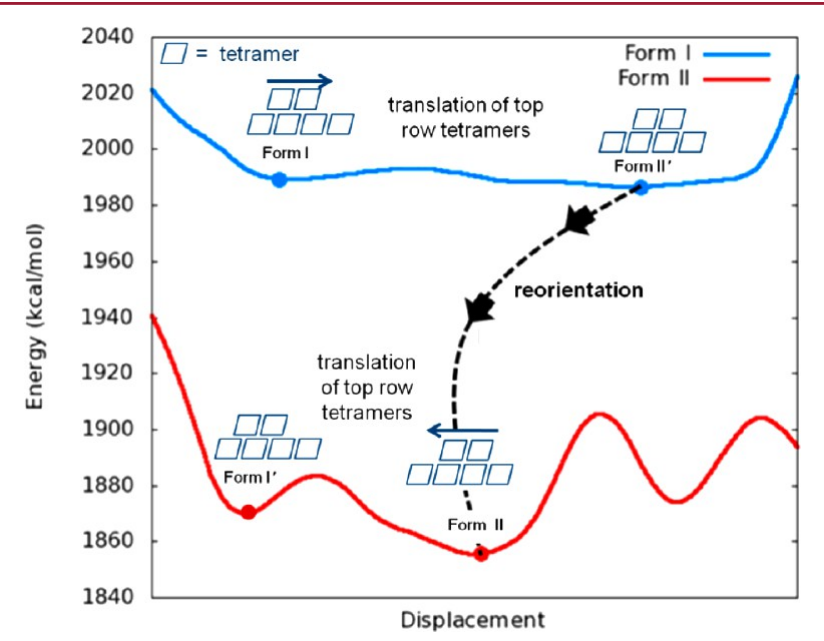

Figure 5. Energy variation along the molecular translation path for the transformation of Form I to II. The blue line indicates the energy profile of moving two tetramers (see the dotted parallelograms in Figure $1 \mathrm{~b}, \mathrm{e}$ ) over another set of four tetramers (Figure S6, Supporting Information), from the equilibrium position of Form I. The position of molecules in the motif at Form II' $^{\prime}$ is nearly similar to that of Form II. A concerted reorientation of packing II' $^{\prime}$ will result in the Form II structure. The bottom red line corresponds to a similar tetramer movement of the Form II motif. Notice that the energy barrier for the movement of top tetramers in Form I is much smaller vis-á-vis than that of the stable Form II, thus explaining the transformation of $\mathbf{I} \rightarrow$ II.

rearrangements will simultaneously replace the weaker $\left(\mathrm{sp}^{3}\right) \mathrm{C}-$ $\mathrm{H} \cdots \mathrm{O}\left(\mathrm{D} / \AA, \theta /{ }^{\circ}: 3.367(3) \AA, 139^{\circ} ; \mathrm{D}=\right.$ distance between heavy atoms, i.e., $\mathrm{C} \cdots \mathrm{O}$ ) interactions (in I) with the slightly stronger bifurcated $\left(\mathrm{sp}^{2}\right) \mathrm{C}-\mathrm{H} \cdots \mathrm{O}\left(3.126(4) \AA, 127^{\circ} ; 3.205 \AA\right.$, $120^{\circ}$ ) interactions (in II). To estimate the potential energy surfaces of these molecular movements in a qualitative manner, we considered a representative motif in the Form I structure (Figure S6a, Supporting Information), where a 1D tape of two tetramers ( 8 molecules) lies above another with four tetramers (16 molecules), and sheared the upper portion until it reached the motif II' $^{\prime}$ (see Figure 5, Figure S6b, Supporting Information), which is close to the packing in Form II. The energies for the corresponding intermediate positions in the path are calculated (blue curve in Figure 5). However, in reality, a concerted readjustment of the molecules while they approach this motif II' shall result in the packing of Form II (Figure S6c, Supporting Information). Similarly, the energy surfaces for the transformation from Form II $\rightarrow$ I are also calculated by moving the upper tape of Form II in the opposite direction (red curve in Figure 5). It is also to be noted that the energy curves shown here do not represent the global structures but are of only a group of molecules within a layer that represent the possible molecular movements at a local level in the phase transition. However, both the curves show that the Form II is more stable than I, which is in good agreement with our experimental finding. It is clear that the barrier for the Form I $\rightarrow$ II transformation is nearly insignificant, indicating a low resistance to conversion from I to II upon stressing, which is in good agreement with the indentation data on (111). Hence, this low energy barrier is the reason for Form I's mechanical instability 
Form I

(a)

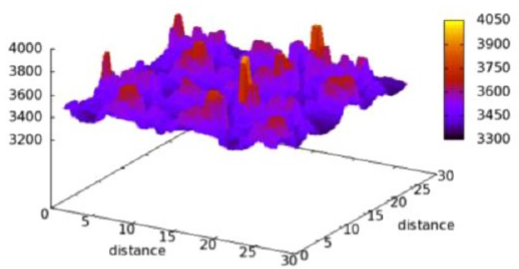

(c)

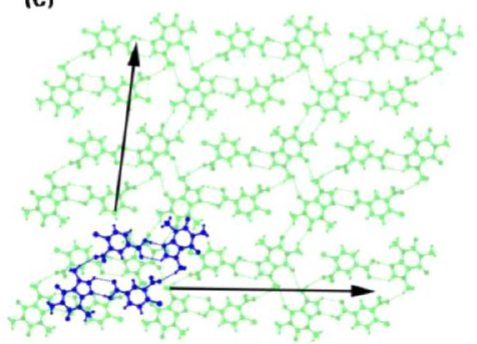

Form II

(b)

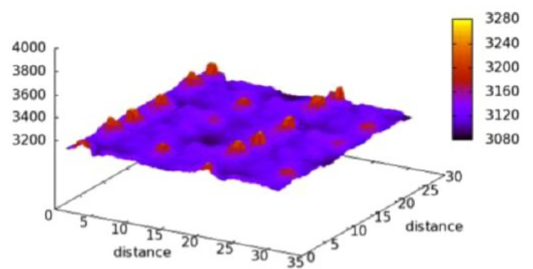

(d)

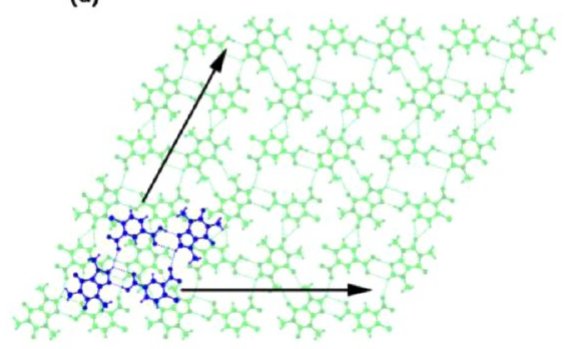

Figure 6. Potential energy surfaces ( $\mathrm{kcal} / \mathrm{mol}$ ) for Forms (a) I and (b) II. The calculations were performed by taking two layers, with one tetrameric motif in the top layer (in blue) and nine tetrameric motifs in the bottom layer (in green) from Form I (c), and shearing type Form II (d). The energy is plotted in the $z$ axis, whereas the $x$ and $y$ axes represent the position of the top layer with respect to the bottom layer. The vector movement of the above tetramer is marked by arrows.

despite its higher hardness. In contrast, a high energy barrier for the reverse direction is noted, implying transformation from Form II (thermodynamically stable form) to I is unfavorable; that is, its layers are much tighter.

We also calculated the energy profile for sliding the layers one over the other, which gave us an estimate of the contributions from $\pi$-stacking interactions (Figure 6). The energy profiles of Forms I and II clearly show that the latter has a smooth potential energy surface (Figure $6 \mathrm{~b}$ ) vis-à-vis the former (Figure 6a). This observation is consistent with the smoother sliding of layers in Form II. This is also in good agreement with the experimentally determined mechanical properties of the two forms; Form $\mathbf{I}$ is harder and brittle and Form II is softer and easy to shear.

The above two calculations provide the qualitative estimate of the contributions from intralayer $\mathrm{C}-\mathrm{H} \cdots \mathrm{O}$ and interlayer $\pi$ stacking interactions separately. However, a question arises, whether the flat potential surface of the $\pi$-stacking forces in Form II is a consequence of its stronger intralayer interactions or a coincidence. It is reasonable to assume that the stronger intralayer interactions demand the alignment of adjacent molecules; this is a restraint for ideal optimization of the stacking contacts in the orthogonal direction, which otherwise requires tilting of aromatic rings. From the hydrogen bond competition studies in crystal engineering, it is also known that the stronger interactions direct the crystal packing, whereas the weaker ones find their idealized positions within the allowed limits. ${ }^{2,20}$ Hence, it is reasonable to assume that the relatively stronger intralayer interactions in Form II limit the optimization of stacking interactions. As a result, the energy surface of stacking forces becomes smoother, which makes it favorable for easy shearing.

\section{CONCLUSIONS}

In conclusion, the results of macroscopic mechanical deformation and computational studies on the two polymorphic co-crystals of 1:1 caffeine and 4-chloro-3-nitrobenzoic acid allowed us to establish the role of weak intralayer $\mathrm{C}-\mathrm{H} \cdots \mathrm{O}$ and $\pi$-stacking (interlayer) interactions, thus their role in determining the mechanical behavior of two structurally similar polymorphs, I and II. Although both the forms have a 2D layer structure, Form I is brittle and shows stress-induced instability due to a low energy transformation barrier and thus can convert readily to Form II upon mechanical grinding. Form II has relatively tighter $2 \mathrm{D}$ sheets, due to more specific intralayer $\left(\mathrm{sp}^{2}\right) \mathrm{C}-\mathrm{H} \cdots \mathrm{O}$ interactions, which restrict the $\pi$-stacking optimization. This, in turn, results in a smooth potential energy surface, which will favor sliding of layers along certain crystallographic directions. The potential energy surface in Form I with relatively weaker $\left(\mathrm{sp}^{3}\right) \mathrm{C}-\mathrm{H} \cdots \mathrm{O}$ intralayer interactions is considerably more corrugated, which offers higher resistance to shearing, and hence, the crystals are brittle. A good agreement between this rationale and the mechanical properties measured with the nanoindentation technique was found with Form I being nearly twice as hard as Form II. Theoretical calculations and the nanoindentation experiments on the (111) face of brittle Form I explain its unusual stressinduced phase transformation to softer Form II. Identification of such sensitive crystal faces in metastable API forms or sensitive explosives can help to improve their mechanical stability by controlling the crystal morphology. Our study on the structurally similar dimorphs underscores the need of considering weak interactions for understanding mechanical behavior, phase transformations, and, in turn, many other associated dynamic phenomena in crystalline molecular materials and hence is important in the context of crystal engineering.

\section{ASSOCIATED CONTENT}

\section{S Supporting Information}

Experimental details, DSC, PXRD, hot-stage microscopy, TGA, including crystallization protocols, crystallographic table, and data for Forms I and II. This material is available free of charge via the Internet at http://pubs.acs.org. Data for Forms I and II 
have been deposited with the Cambridge Crystallographic Data Centre, CCDC reference numbers 900343 and 900342.

\section{AUTHOR INFORMATION}

\section{Corresponding Authors}

*E-mail: cmallareddy@gmail.com (C.M.R.).

*E-mail: ramu@materials.iisc.ernet.in (U.R.).

\section{Notes}

The authors declare no competing financial interest.

\section{ACKNOWLEDGMENTS}

S.G. thanks the CSIR (New Delhi) for SRF. C.M.R. is grateful for financial support from the DST (SR/FT/CS-074/2009).

\section{REFERENCES}

(1) Desiraju, G. R. Crystal Engineering: The Design of Organic Solids; Elsevier: New York, 1989.

(2) (a) Desiraju, G. R. Angew. Chem., Int. Ed. 2007, 46, 8342-8356. (b) Desiraju, G. R.; Steiner, T. The Weak Hydrogen Bond in Structural Chemistry and Biology; Oxford University Press: Oxford, UK, 1999.

(3) (a) Varughese, S.; Kiran, M. S. R. N.; Solanko, K. A.; Bond, A. D.; Ramamurty, U.; Desiraju, G. R. Chem. Sci. 2011, 2, 2236-2242. (b) Varughese, S.; Kiran, M. S. R. N.; Ramamurty, U.; Desiraju, G. R. Angew. Chem., Int. Ed. 2012, 51, 2-14. (c) Varughese, S.; Kiran, M. S. R. N.; Ramamurty, U.; Desiraju, G. R. Chem.-Asian J. 2012, 7, 21182125. (d) Kiran, M. S. R. N.; Varughese, S.; Reddy, C. M.; Ramamurty, U.; Desiraju, G. R. Cryst. Growth Des. 2010, 10, 4650-4655. (e) Karunatilaka, C.; Bučar, D.-K.; Ditzler, L. R.; Friščić, T.; Swenson, D. C.; MacGillivray, L. R.; Tivanski, A. V. Angew. Chem., Int. Ed. 2011, 50, 8642-8646. (f) Karki, S.; Friščić, T.; Fábián, L.; Laity, P. R.; Day, G. M.; Jones, W. Adv. Mater. 2009, 21, 3905-3909. (g) Fabbiani, F. P. A.; Allan, D. R.; David, W. I. F.; Davidson, A. J.; Lennie, A. R.; Parsons, S.; Pulham, C. R.; Warren, J. E. Cryst. Growth Des. 2007, 7, 1115-1124.

(4) (a) Kelly, A.; Macmillan, N. H. Strong Solids, 3rd ed.; Oxford University Press: Oxford, UK, 1987. (b) Zhang, G. G. Z.; Gu, C.; Zell, M. T.; Burkhardt, R. T.; Munson, E. J.; Grant, D. J. W. J. Pharm. Sci. 2002, 91, 1089-1100. (c) Reddy, C. M.; Krishna, G. R.; Ghosh, S. CrystEngComm 2010, 2296-2314. (d) Ghosh, S.; Reddy, C. M. Angew. Chem., Int. Ed. 2012, 51, 10319-10323. (e) Krishna, G. R.; Kiran, M. S. R. N.; Fraser, C. L.; Ramamurty, U.; Reddy, C. M. Adv. Funct. Mater. 2013, 23, 1422. (f) Zhang, C.; Wang, X.; Huang, H. J. Am. Chem. Soc. 2008, 130, 8359-8365. (g) Munday, L. B.; Solares, S. D.; Chung, P. W. Philos. Mag. 2012, 92, 3036-3050.

(5) (a) Naumov, P.; Kowalik, J.; Solntsev, K. M.; Baldridge, A.j Moon, J.-S.; Kranz, C.; Tolbert, L. M. J. Am. Chem. Soc. 2010, 132, 5845-5857. (b) John, G.; Jadhav, S. R.; Menon, V. M.; John, V. T. Angew. Chem., Int. Ed. 2012, 51, 1760-1762. (c) Fratzl, P.; Barth, F. G. Nature 2009, 462, 442-448.

(6) (a) Bernstein, J. Polymorphism in Molecular Crystals; Oxford University Press: Oxford, U.K., 2002. (b) Davey, R. J. Chem. Commun. 2003, 1463. (c) Byrn, S. R.; Pfeiffer, R. R; Stowell, J. G. Solid-State Chemistry of Drugs; SSCI: West Lafayette, IN, 1999. (d) Brittain, H. G. Polymorphism in Pharmaceutical Solids; Marcel Dekker: New York, 1999. (e) Hilfiker, R. Polymorphism in the Pharmaceutical Industry; Wiley: Weinheim, 2006. (f) Boldyreva, E. V. J. Mol. Struct. 2003, 647, 159 and references therein. (g) Fabbiani, F. P. A.; Pulham, C. R. Chem. Soc. Rev. 2006, 35, 932 and references therein. (h) Bordallo, H. N.; Boldyreva, E. V.; Buchsteiner, A.; Koza, M. M.; Landsgesell, S. J. Phys. Chem. 2008, 112, 8748.

(7) (a) Braga, D.; Grepioni, F. Angew. Chem., Int. Ed. 2004, 43, 4002. (b) Trask, A. V.; Motherwell, W. D. S.; Jones, W. Chem. Commun. 2002, 2372. (c) Friščić, T.; Jones, W. Cryst. Growth Des. 2009, 9, 1621. (d) Bag, P. P.; Chen, M.; Sun, C. C.; Reddy, C. M. CrystEngComm 2012, 14, 3865-3867. (e) Sun, C. C.; Hou, H. Cryst. Growth Des. 2008, 8, 1575. (f) Sun, C. C.; Grant, D. J. W. Pharm. Res. 2001, 18, 281-286. (g) Sun, C. C. J. Pharm. Sci. 2009, 98, 1671.
(8) (a) SAINT Plus (version 6.45); Bruker AXS Inc.: Madison, WI, 2003. (b) SMART (version 5.625) and SHELX-TL (version 6.12); Bruker AXS Inc.: Madison, WI, 2000.

(9) Barbour, L. J. X-Seed, Graphical Interface to SHELX-97 and POVRay; University of Missouri: Columbia, MO, 1999.

(10) Oliver, W. C.; Pharr, G. M. J. Mater. Res. 1992, 7, 1564-1583.

(11) Bolshakov, A.; Oliver, W. C.; Pharr, G. M. J. Mater. Res. 1996, 11, 760-768.

(12) (a) Ramos, K. J.; Bahr, D. F. J. Mater. Res. 2007, 22, 2037-2045. (b) Ramos, K. J.; Hooks, D. E.; Bahr, D. F. Philos. Mag. 2009, 89, 2381-2402.

(13) Roy, S.; Bhatt, P. M.; Nangia, A.; Kruger, G. J. Cryst. Growth Des. 2007, 3, 476-480.

(14) Espeau, P.; Céolin, R.; Tamarit, J.-L.; Perrin, M.-A.; Gauchi, J.P.; Leveiller, F. J. Pharm. Sci. 2005, 94, 524-539.

(15) Shishkin, O. V.; Medvediev, V. V.; Zubatyuk, R. I. CrystEngComm 2013, 15, 160-167.

(16) Casellas, D.; Caro, J.; Kolas, S.; Prado, J. M.; Valls, I. Acta Mater. 2007, 55, 4277-4286.

(17) Yoo, B.-G.; Park, K.-W.; Lee, J.-C.; Ramamurty, U.; Jang, J.-I. J. Mater. Res. 2009, 24, 1405-1416.

(18) Bahr, D. F.; Kramer, D. E.; Gerberich, W. W. Acta Mater. 1998 46, 3605-3617.

(19) (a) Dowling, R.; Davey, R. J.; Curtis, R. A.; Han, G.; Poornachary, S. K.; Chow, P. S.; Tan, R. B. H. Chem. Commun. 2010, 46, 5924-5926. (b) Banerjee, S.; Adarsh, N. N.; Dastidar, P. CrystEngComm 2013, 15, 245-248.

(20) Etter, M. C. Acc. Chem. Res. 1990, 23, 120-126. 